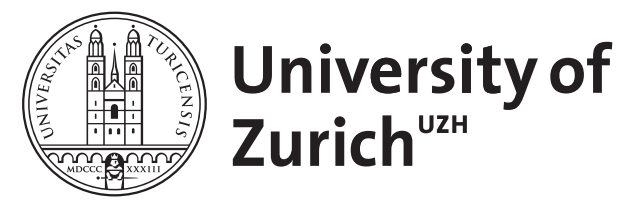

\title{
Higgs boson production at hadron colliders: hard-collinear coefficients at the NNLO
}

\author{
Catani, Stefano ; Grazzini, Massimiliano
}

\begin{abstract}
We consider the production of the Standard Model Higgs boson through the gluon fusion mechanism in hadron collisions. We present the next-to-next-to-leading order (NNLO) QCD result of the hard-collinear coefficient function for the all-order resummation of logarithmically enhanced contributions at small transverse momentum. The coefficient function controls NNLO contributions in resummed calculations at full next-to-next-to-leading logarithmic accuracy. The same coefficient function is used in applications of the subtraction method to perform fully exclusive perturbative calculations up to NNLO.
\end{abstract}

DOI: https://doi.org/10.1140/epjc/s10052-012-2013-2

Posted at the Zurich Open Repository and Archive, University of Zurich ZORA URL: https://doi.org/10.5167/uzh-70197

Journal Article

Originally published at:

Catani, Stefano; Grazzini, Massimiliano (2012). Higgs boson production at hadron colliders: hardcollinear coefficients at the NNLO. European Physical Journal C - Particles and Fields, 72(5):online. DOI: https://doi.org/10.1140/epjc/s10052-012-2013-2 
ZU-TH 12/11

\title{
Higgs boson production at hadron colliders: hard-collinear coefficients at the NNLO
}

\author{
Stefano Catani $^{(a)}$ and Massimiliano Grazzini ${ }^{(b) *}$ \\ (a) INFN, Sezione di Firenze and Dipartimento di Fisica e Astronomia, \\ Università di Firenze, I-50019 Sesto Fiorentino, Florence, Italy \\ (b) Institut für Theoretische Physik, Universität Zürich, CH-8057 Zürich, Switzerland
}

\begin{abstract}
We consider the production of the Standard Model Higgs boson through the gluon fusion mechanism in hadron collisions. We present the next-to-next-to-leading order (NNLO) QCD result of the hard-collinear coefficient function for the allorder resummation of logarithmically-enhanced contributions at small transverse momentum. The coefficient function controls NNLO contributions in resummed calculations at full next-to-next-to-leading logarithmic accuracy. The same coefficient function is used in applications of the subtraction method to perform fully-exclusive perturbative calculations up to NNLO.
\end{abstract}

June 2011

*On leave of absence from INFN, Sezione di Firenze, Sesto Fiorentino, Florence, Italy. 
The transverse-momentum $\left(q_{T}\right)$ distribution of systems with high invariant mass $M$ (such as Drell-Yan lepton pairs, photon pairs, vector bosons, Higgs bosons, and so forth) produced in hadron collisions is computable by using perturbative QCD. However, in the small- $q_{T}$ region (roughly, in the region where $q_{T} \ll M$ ) the convergence of the fixed-order perturbative expansion in powers of the QCD coupling $\alpha_{\mathrm{S}}$ is spoiled by the presence of large logarithmic contributions of the type $\ln ^{n}\left(M^{2} / q_{T}^{2}\right)$. The predictivity of perturbative QCD can be recovered through the summation of these logarithmically-enhanced contributions to all order in $\alpha_{\mathrm{S}}$ [1].

The structure of the resummed calculation can be organized in a process-independent form $[2,3,4,5]$, in which the logarithmic contributions are controlled by a set of perturbative functions, usually denoted as $A\left(\alpha_{\mathrm{S}}\right), B\left(\alpha_{\mathrm{S}}\right), C\left(\alpha_{\mathrm{S}}\right)$ and $\mathcal{H}\left(\alpha_{\mathrm{S}}\right)$ (see, e.g., Eqs. (8) and (16) and related comments). These functions and, hence, their perturbative coefficients (e.g. the coefficient $A^{(n)}$ of the $n$-th order contribution $A^{(n)} \alpha_{\mathrm{S}}^{n}$ to $A\left(\alpha_{\mathrm{S}}\right)$ ), have no explicit dependence on the ratio $q_{T} / M$. The perturbative coefficients, once they are known, can be inserted in process-independent resummation formulae that systematically resum, in explicit form, the classes of leading, next-to-leading, next-to-next-to-leading (and so forth) logarithmic contributions to the transverse-momentum distribution. In this respect, the transverse-momentum resummation program has formal analogies ${ }^{\dagger}$ with the study of logarithmic scaling violations (of ultraviolet or collinear origin), where the resummation of logarithmic terms is traded for the calculation of perturbative functions, such as short-distance coefficient functions and anomalous dimensions.

Most of the $q_{T}$ resummation coefficients are known, since some time $[6,7,8,9]$, up to the second order in $\alpha_{\mathrm{S}}$. The third-order coefficient $A^{(3)}$ has been obtained in Ref. [10]. In recent years, we have been working on a research project aimed at the completion of the $q_{T}$ resummation program at the second perturbative order. This requires the calculation of the second-order coefficient function $\mathcal{H}^{(2)}(z)$ (see Eq. (19)), which includes a process-dependent part. The computation of the $\mathcal{H}^{(2)}$ coefficients has been explicitly carried out for two benchmark processes, namely, Higgs boson production and the Drell-Yan process, and the corresponding results have been obtained and used in Refs. [11] and [12], respectively. In the case of the Drell-Yan process, the result has also been applied [13] to the $q_{T}$ spectrum of the $Z$ boson, by explicitly performing transversemomentum resummation at the next-to-next-to-leading logarithmic (NNLL) accuracy. In this paper, we consider Higgs boson production and we document the result for $\mathcal{H}^{(2)}$ [11] in explicit analytic form. We also illustrate the method that we have used to perform the calculation.

Considering the perturbative contributions that are logarithmically-enhanced and, thus, singular in the limit $q_{T} \rightarrow 0$, the calculation of $\mathcal{H}^{(2)}$ completes the knowledge of the $q_{T}$ distribution at full next-to-next-to-leading order (NNLO) accuracy. This fact has implications in the context of both resummed and fixed-order calculations, as we briefly discuss below.

Some recent resummed calculations of the $q_{T}$ spectrum of the Standard Model (SM) Higgs boson at Tevatron and LHC energies are presented in Refs. [14]-[21]. The inclusion of $\mathcal{H}^{(2)}$ in calculations that use the other $q_{T}$ resummation coefficients up to NNLL order gives theoretical predictions that embody the exact NNLO calculation in the small- $q_{T}$ region. The NNLL resummed calculations can then be properly matched (by using, for instance, $q_{T}$ resummation as in the

\footnotetext{
${ }^{\dagger}$ These analogies may hide important physical, conceptual and technical differences, which are discussed in the literature on transverse-momentum resummation.

${ }^{\ddagger}$ In this introductory part we are using a shorthand notation, since the symbol $\mathcal{H}^{(2)}$ actually refers to a set of several coefficient functions.
} 
formalism of Ref. [19]) with the customary fixed-order calculation at large $q_{T}$, in such a way that the integration over $q_{T}$ of the $q_{T}$ distribution exactly returns the NNLO value of the total cross section. Indeed, a rough approximation of $\mathcal{H}^{(2)}$, such as to reproduce the NNLO value of the total cross section with good numerical accuracy, was constructed and used in Ref. [19]. The approximation of Ref. [19] represents a very crude estimate of the function $\mathcal{H}^{(2)}(z)$; nonetheless, that approximation quantitatively works very well (especially at LHC energies) $[19,20]$ over a wide range of Higgs boson masses. An updated version of the code HqT [19], which also implements the exact coefficient $\mathcal{H}^{(2)}$, is now available [22].

In Ref. [11], we presented a practical formalism to perform NNLO calculations at the fullyexclusive level for a specific class of processes, namely, the production of colourless high-mass systems in hadron collisions. The formalism exploits the subtraction method to cancel the unphysical infrared divergences that separately occur in the real and virtual radiative corrections. The explicit construction of the subtraction counterterms $[19,20]$ is based on the process-independent structure of transverse-momentum resummation formulae and on their expansion up to NNLO in QCD perturbation theory. The formalism thus requires the complete knowledge of the $q_{T}$ resummation coefficients up to $\mathcal{O}\left(\alpha_{\mathrm{S}}^{2}\right)$. Although the results of the present paper were not explicitly illustrated in Ref. [11], they were taken into account in the NNLO computations presented therein. In particular, the explicit application to Higgs boson production (which was implemented in the Monte Carlo code HNNLO) considered in Refs. [11, 23] is based on and implements the analytic results for the coefficient function $\mathcal{H}^{(2)}$ that are documented in the present paper.

The paper is organized as follows. We first introduce our notation and describe the small$q_{T}$ behaviour of the Higgs boson cross section up to NNLO. Then we briefly review transversemomentum resummation for Higgs boson production and the corresponding all-order resummation formula recently derived in Ref. [5]. The new resummation formula differs from its naïve version that is used in the literature: the differences start at $\mathcal{O}\left(\alpha_{\mathrm{S}}^{2}\right)$, which is relevant for the purposes of the present paper. Finally, we present the analytic results of our NNLO calculation of the $q_{T}$ distribution. The results are expressed directly in terms of the coefficient function $\mathcal{H}^{(2)}(z)$ and related resummation coefficients. We conclude the paper by describing the method that we have used to perform the NNLO calculation.

We briefly introduce the theoretical framework and our notation. We consider the production of the SM Higgs boson $H$, through the gluon fusion mechanism $g g \rightarrow H$, in hadron-hadron collisions. The effective coupling $g g H$ is produced by heavy-quark loops, and the top quark gives the dominant contribution. We treat the coupling $g g H$ in the framework of the large- $m_{t o p}$ approximation $[24,25,26]$, and we consider a single heavy quark, the top quark with mass $m_{t o p}$, and $n_{F}\left(n_{F}=5\right)$ massless-quark flavours. We use the narrow width approximation and we treat the Higgs boson as an on-shell particle with mass $M$. The QCD expression of the Higgs boson transverse-momentum cross section is

$$
\frac{d \sigma}{d q_{T}^{2}}\left(q_{T}, M, s\right)=\sum_{a, b} \int_{0}^{1} d z_{1} \int_{0}^{1} d z_{2} f_{a / h_{1}}\left(z_{1}, M^{2}\right) f_{b / h_{2}}\left(z_{2}, M^{2}\right) \frac{d \hat{\sigma}_{a b}}{d q_{T}^{2}}\left(q_{T}, M, \hat{s}=z_{1} z_{2} s ; \alpha_{\mathrm{S}}\left(M^{2}\right)\right)
$$

where $f_{a / h_{i}}\left(x, \mu_{F}^{2}\right)\left(a=q_{f}, \bar{q}_{f}, g\right)$ are the parton densities of the colliding hadrons $\left(h_{1}\right.$ and $\left.h_{2}\right)$ at the factorization scale $\mu_{F}$, and $d \hat{\sigma}_{a b} / d q_{T}^{2}$ are the partonic cross sections. The centre-of-mass energy of the two colliding hadrons is denoted by $s$, and $\hat{s}$ is the partonic centre-of-mass energy. We use parton densities as defined in the $\overline{\mathrm{MS}}$ factorization scheme, and $\alpha_{\mathrm{S}}\left(\mu_{R}^{2}\right)$ is the QCD running 
coupling at the renormalization scale $\mu_{R}$ in the $\overline{\mathrm{MS}}$ renormalization scheme. In Eq. (1) and throughout the paper, the arbitrary factorization and renormalization scales, $\mu_{F}$ and $\mu_{R}$, are set to be equal to the Higgs boson mass $M$.

The partonic cross sections $d \hat{\sigma}_{a b} / d q_{T}^{2}$ are computable in QCD perturbation theory as power series expansions in $\alpha_{\mathrm{S}}\left(M^{2}\right)$. We are interested in the perturbative contributions that are large in the small- $q_{T}$ region $\left(q_{T} \ll M\right)$ and, eventually, singular in the limit $q_{T} \rightarrow 0$. To explicitly present the perturbative structure of these enhanced terms at small $q_{T}$, we integrate the $q_{T}$ distribution over the region $0 \leq q_{T} \leq Q_{0}$, and we introduce the cumulative partonic cross section

$$
\int_{0}^{Q_{0}^{2}} d q_{T}^{2} \frac{d \hat{\sigma}_{a b}}{d q_{T}^{2}}\left(q_{T}, M, \hat{s}=M^{2} / z ; \alpha_{\mathrm{S}}\left(M^{2}\right)\right) \equiv z \sigma_{H}^{(0)}\left(\alpha_{\mathrm{S}}\left(M^{2}\right)\right) \hat{R}_{a b}\left(z, M / Q_{0} ; \alpha_{\mathrm{S}}\left(M^{2}\right)\right)
$$

where the overall normalization of the function $\hat{R}_{a b}$ is defined with respect to $\sigma_{H}^{(0)}$, which is the Born level cross section for the partonic subprocess $g g \rightarrow H$. Using the large- $m_{t o p}$ approximation, the explicit expression of $\sigma_{H}^{(0)}$ is [24]

$$
\sigma_{H}^{(0)}\left(\alpha_{\mathrm{S}}\right)=\frac{G_{F} \alpha_{\mathrm{S}}^{2}}{288 \pi \sqrt{2}}
$$

where $G_{F}$ is the Fermi constant. The partonic function $\hat{R}$ has the following perturbative expansion

$$
\hat{R}_{a b}\left(z, M / Q_{0} ; \alpha_{\mathrm{S}}\right)=\delta_{g a} \delta_{g b} \delta(1-z)+\sum_{n=1}^{\infty}\left(\frac{\alpha_{\mathrm{S}}}{\pi}\right)^{n} \hat{R}_{a b}^{(n)}\left(z, M / Q_{0}\right)
$$

The next-to-leading order (NLO) and NNLO contributions to the cumulative cross section in Eq. (2) are determined by the functions $\hat{R}^{(1)}$ and $\hat{R}^{(2)}$, respectively. The small- $q_{T}$ region of the cross section $d \hat{\sigma}_{a b} / d q_{T}^{2}$ is probed by performing the limit $Q_{0} \ll M$ in Eq. (2). In this limit, the NLO and NNLO functions $\hat{R}^{(1)}$ and $\hat{R}^{(2)}$ have the following behaviour:

$$
\begin{gathered}
\hat{R}_{a b}^{(1)}\left(z, M / Q_{0}\right)=l_{0}^{2} \hat{R}_{a b}^{(1 ; 2)}(z)+l_{0} \hat{R}_{a b}^{(1 ; 1)}(z)+\hat{R}_{a b}^{(1 ; 0)}(z)+\mathcal{O}\left(Q_{0}^{2} / M^{2}\right) \\
\hat{R}_{a b}^{(2)}\left(z, M / Q_{0}\right)=l_{0}^{4} \hat{R}_{a b}^{(2 ; 4)}(z)+l_{0}^{3} \hat{R}_{a b}^{(2 ; 3)}(z)+l_{0}^{2} \hat{R}_{a b}^{(2 ; 2)}(z)+l_{0} \hat{R}_{a b}^{(2 ; 1)}(z)+\hat{R}_{a b}^{(2 ; 0)}(z)+\mathcal{O}\left(Q_{0}^{2} / M^{2}\right)
\end{gathered}
$$

where $l_{0}=\ln \left(M^{2} / Q_{0}^{2}\right)$. In Eqs. (5) and (6), the powers of the large logarithm $l_{0}$ are produced by the singular (though, integrable) behaviour of $d \hat{\sigma}_{a b} / d q_{T}^{2}$ at small values of $q_{T}$. The coefficients $\hat{R}^{(1 ; m)}$ (with $m \leq 2$ ) and $\hat{R}^{(2 ; m)}$ (with $m \leq 4$ ) of the large logarithms are independent of $Q_{0}$; these coefficients depend on the partonic centre-of-mass energy $\hat{s}$ and, more precisely, they are functions of the energy fraction $z=M^{2} / \hat{s}$.

In this paper we present the computation of the cumulative cross section in Eq. (2) up to NNLO. The partonic calculation is performed in analytic form by neglecting terms of $\mathcal{O}\left(Q_{0}^{2} / M^{2}\right)$ in the limit $Q_{0} \ll M$. Therefore, we determine the coefficient functions $\hat{R}^{(n ; m)}(z)$ in Eqs. (5) and (6). Before presenting the results, we illustrate how these functions are related to the perturbative coefficients of the transverse-momentum resummation formula for Higgs boson production [3, 5]. This relation, which allows us to extract the $q_{T}$ resummation coefficients up to $\mathcal{O}\left(\alpha_{\mathrm{S}}^{2}\right)$, also shows 
that the knowledge of Eq. (6) is sufficient to fully determine the NNLO rapidity distribution of the Higgs boson in the small- $q_{T}$ region.

The partonic cross section $d \hat{\sigma}_{a b} / d q_{T}^{2}$ in Eq. (1) can be decomposed in the form $d \hat{\sigma}_{a b}=d \hat{\sigma}_{a b}^{\text {(sing) }}+$ $d \hat{\sigma}_{a b}^{(\mathrm{reg})}$. The singular component, $d \hat{\sigma}_{a b}^{(\mathrm{sing})}$, contains all the contributions that are enhanced at small $q_{T}$. These contributions are proportional to $\delta\left(q_{T}^{2}\right)$ or to large logarithms ${ }^{\S}$ of the type $\frac{1}{q_{T}^{2}} \ln ^{m}\left(M^{2} / q_{T}^{2}\right)$. On the contrary, the remaining component, $d \hat{\sigma}_{a b}^{(\mathrm{reg})}$, of the partonic cross section is regular order-by-order in $\alpha_{\mathrm{S}}$ as $q_{T} \rightarrow 0$. To be precise, the integration of $d \hat{\sigma}_{a b}^{(\mathrm{reg})} / d q_{T}^{2}$ over the range $0 \leq q_{T} \leq Q_{0}$ leads to a finite result that, at each fixed order in $\alpha_{\mathrm{S}}$, vanishes in the limit $Q_{0} \rightarrow 0$. Therefore, $d \hat{\sigma}_{a b}^{(\mathrm{reg})}$ only contributes to the terms of $\mathcal{O}\left(Q_{0}^{2} / M^{2}\right)$ on the right-hand side of Eqs. (5) and (6). The decomposition of the partonic cross sections can be inserted in the right-hand side of Eq. (1), thus leading to the corresponding decomposition of the hadronic cross section $d \sigma / d q_{T}^{2}$, namely, $d \sigma=d \sigma^{(\text {sing })}+d \sigma^{(\text {reg })}$.

We consider the singular component of the Higgs boson $q_{T}$ cross section, and we recall its all-order perturbative structure. We directly refer to the hadronic cross section (rather than the partonic cross sections), since its structure can be presented by using a more compact notation. Moreover, to illustrate the general kinematics of transverse-momentum resummation, we consider the $q_{T}$ cross section $d \sigma / d y d q_{T}^{2}$ at fixed value of the rapidity $y$ of the Higgs boson (the rapidity is defined in the centre-of-mass frame of the two colliding hadrons). The transverse-momentum resummation formula for the singular component of the Higgs boson cross section is [3, 5]

$$
\begin{aligned}
& \frac{d \sigma^{(\text {sing })}}{d y d q_{T}^{2}}\left(y, q_{T}, M, s\right)=\frac{M^{2}}{s} \sigma_{H}^{(0)}\left(\alpha_{\mathrm{S}}\left(M^{2}\right)\right) \int_{0}^{+\infty} d b \frac{b}{2} J_{0}\left(b q_{T}\right) S_{g}(M, b) \\
& \quad \times \sum_{a_{1}, a_{2}} \int_{x_{1}}^{1} \frac{d z_{1}}{z_{1}} \int_{x_{2}}^{1} \frac{d z_{2}}{z_{2}}\left[H^{F} C_{1} C_{2}\right]_{g g ; a_{1} a_{2}} f_{a_{1} / h_{1}}\left(x_{1} / z_{1}, b_{0}^{2} / b^{2}\right) f_{a_{2} / h_{2}}\left(x_{2} / z_{2}, b_{0}^{2} / b^{2}\right),
\end{aligned}
$$

where the kinematical variables $x_{i}(i=1,2)$ are $x_{1}=e^{+y} M / \sqrt{s}$ and $x_{2}=e^{-y} M / \sqrt{s}$. The integration variable $b$ is the impact parameter, $J_{0}\left(b q_{T}\right)$ is the 0th-order Bessel function, and $b_{0}=2 e^{-\gamma_{E}}$ $\left(\gamma_{E}=0.5772 \ldots\right.$ is the Euler number $)$ is a numerical coefficient. The symbol $\left[H^{F} C_{1} C_{2}\right]_{g g ; a_{1} a_{2}}$ denotes (see Eq. (44) in Ref. [5]) the following function of the longitudinal-momentum fractions $z_{1}$ and $z_{2}$ :

$$
\begin{aligned}
{\left[H^{H} C_{1} C_{2}\right]_{g g ; a_{1} a_{2}}=H_{g}^{H}\left(\alpha_{\mathrm{S}}\left(M^{2}\right)\right) } & {\left[C_{g a_{1}}\left(z_{1} ; \alpha_{\mathrm{S}}\left(b_{0}^{2} / b^{2}\right)\right) C_{g a_{2}}\left(z_{2} ; \alpha_{\mathrm{S}}\left(b_{0}^{2} / b^{2}\right)\right)\right.} \\
& \left.+G_{g a_{1}}\left(z_{1} ; \alpha_{\mathrm{S}}\left(b_{0}^{2} / b^{2}\right)\right) G_{g a_{2}}\left(z_{2} ; \alpha_{\mathrm{S}}\left(b_{0}^{2} / b^{2}\right)\right)\right]
\end{aligned}
$$

where $H_{g}^{H}\left(\alpha_{\mathrm{S}}\right), C_{g a}\left(z ; \alpha_{\mathrm{S}}\right)$ and $G_{g a}\left(z ; \alpha_{\mathrm{S}}\right)$ are perturbative functions of $\alpha_{\mathrm{S}}$ (see Eqs. (9)-(11)). The other perturbative ingredients of Eq. (7) are the function $S_{g}(M, b)$, which is the Sudakov form factor of the gluon (see the comments below), and $\sigma_{H}^{(0)}\left(\alpha_{\mathrm{S}}\left(M^{2}\right)\right)$, which is the Born level cross section in Eq. (3).

The structure of Eq. (7) is well known in the literature on resummed calculations for the $q_{T}$ spectrum of the Higgs boson. However, the functional form of Eq. (8) is new [5]. The customary

$\S^{\S}$ To be precise, the logarithms are combined with corresponding 'contact' terms, which are proportional to $\delta\left(q_{T}^{2}\right)$. These combinations define regularized (integrable) 'plus distributions' $\left[\frac{1}{q_{T}^{2}} \ln ^{m}\left(M^{2} / q_{T}^{2}\right)\right]_{+}$with respect to $q_{T}^{2}$. The cumulative cross section in Eq. (2) is insensitive to the precise mathematical definition of these 'plus distributions'. 
'naïve' version (i.e. the version extrapolated from the transverse-momentum resummation formula for the Drell-Yan process) of Eq. (8) includes only the perturbative functions $H_{g}^{H}$ and $C_{g a}$. The presence of an additional term, due to the function $G_{g a}$, has been pointed out in Ref. [5]. Note that the function $G_{g_{a}}\left(z ; \alpha_{\mathrm{S}}\right)$ is of $\mathcal{O}\left(\alpha_{\mathrm{S}}\right)$ (see Eq. (9)) and, therefore, it leads to a contribution of $\mathcal{O}\left(\alpha_{\mathrm{S}}^{2}\right)$ in the right-hand side of Eq. (8). This fact implies that the presence of $G_{g a}\left(z ; \alpha_{\mathrm{S}}\right)$ cannot be detected through a NLO calculation of the $q_{T}$ spectrum of the Higgs boson. This fact also implies that our NNLO analytic calculation of the cumulative cross section in Eq. (2) gives an explicit check of the presence of $G_{g a}\left(z ; \alpha_{\mathrm{S}}\right)$ and of its precise form at $\mathcal{O}\left(\alpha_{\mathrm{S}}\right)$ (see Eq. (28) and related comments).

The gluon form factor $S_{g}(M, b)$ of Eq. (7) is a process-independent quantity [3, 8, 4]. Its functional dependence on $M$ and $b$ is controlled by two perturbative functions, which are usually denoted as $A_{g}\left(\alpha_{\mathrm{S}}\right)$ and $B_{g}\left(\alpha_{\mathrm{S}}\right)$ (see, e.g., Eqs. (10)-(12) in Ref. [5]). Their corresponding $n$-th order perturbative coefficients are $A_{g}^{(n)}$ and $B_{g}^{(n)}$. The coefficients $A_{g}^{(1)}, B_{g}^{(1)}, A_{g}^{(2)}[8]$ and $B_{g}^{(2)}[9]$ are known: their knowledge fully determines the perturbative expression of $S_{g}(M, b)$ up to $\mathcal{O}\left(\alpha_{\mathrm{S}}^{2}\right)$.

The quantity $\left[H^{F} C_{1} C_{2}\right]$ in Eq. (7) depends on the three perturbative functions $H_{g}^{H}, C_{g a}$ and $G_{g_{a}}$. By inspection of the right-hand side of Eq. (8), we notice that the scale of $\alpha_{\mathrm{S}}$ is not set to a unique value. We have $\alpha_{\mathrm{S}}\left(M^{2}\right)$ in the case of the function $H_{g}^{H}\left(\alpha_{\mathrm{S}}\right)$, and $\alpha_{\mathrm{S}}\left(b_{0}^{2} / b^{2}\right)$ in the case of the functions $C_{g a}\left(\alpha_{\mathrm{S}}\right)$ and $G_{g a}\left(\alpha_{\mathrm{S}}\right)$. The presence of these two different arguments of $\alpha_{\mathrm{S}}$ is related to the physical origin $[4,5]$ of the corresponding perturbative functions. Roughly speaking, $H_{g}^{H}\left(\alpha_{\mathrm{S}}\left(M^{2}\right)\right)$ embodies contributions due to the hard-momentum region ${ }^{\dagger}$ of the virtual corrections to the lowest-order subprocess $g g \rightarrow H$. The functions $C_{g a}$ and $G_{g a}$ instead refer to the inclusive subprocess $g a \rightarrow H+X$ : roughly speaking, $C_{g a}\left(\alpha_{\mathrm{S}}\left(b_{0}^{2} / b^{2}\right)\right)$ and $G_{g a}\left(\alpha_{\mathrm{S}}\left(b_{0}^{2} / b^{2}\right)\right)$ originate from the kinematical region where the momenta of the partons in the final-state system $X$ are (almost) collinear to the momentum of the initial-state parton $a$. Owing to this physical picture, the quantity $\left[H^{F} C_{1} C_{2}\right]$ can be regarded as a hard-collinear partonic function. Note that the function $H_{g}^{H}\left(\alpha_{\mathrm{S}}\right)$ is process dependent, since it is directly related to the production mechanism of the SM Higgs boson. On the contrary, the partonic functions $C_{g a}$ and $G_{g a}$ are process independent, as a consequence of the universality features of QCD collinear radiation.

We recall that the functions $H_{g}^{H}\left(\alpha_{\mathrm{S}}\right), C_{g a}\left(\alpha_{\mathrm{S}}\right), G_{g a}\left(\alpha_{\mathrm{S}}\right)$ and the perturbative function $B_{g}\left(\alpha_{\mathrm{S}}\right)$ of the gluon form factor are not separately computable in an unambiguous way. Indeed, these four functions are correlated (constrained) by a renormalization-group symmetry [4] that is related to the $b$-space factorization structure of Eqs. (7) and (8). The unambiguous definition of these four functions thus requires the specification ${ }^{\ddagger}$ of a resummation scheme [4]. Note, however, that considering the perturbative expansion ${ }^{\S}$ of Eq. (7) (i.e., the perturbative expansion of the singular component of the $q_{T}$ cross section), the resummation-scheme dependence exactly cancels orderby-order in $\alpha_{S}$.

The perturbative expansion of the three functions on the right-hand side of Eq. (8) is defined

\footnotetext{
†This is the region where the size of the momenta of the virtual loops is of the order of $M$.

¥The reader who is not interested in issues related to the specification of a resummation scheme can simply assume that $H_{g}^{H}\left(\alpha_{\mathrm{S}}\right) \equiv 1$ throughout this paper.

$\S$ The resummation-scheme dependence also cancels by consistently expanding Eq. (7) in terms of classes of resummed (leading, next-to-leading and so forth) logarithmic contributions [19].
} 
as follows:

$$
\begin{gathered}
G_{g a}\left(z ; \alpha_{\mathrm{S}}\right)=\frac{\alpha_{\mathrm{S}}}{\pi} G_{g a}^{(1)}(z)+\sum_{n=2}^{\infty}\left(\frac{\alpha_{\mathrm{S}}}{\pi}\right)^{n} G_{g a}^{(n)}(z) \\
C_{g a}\left(z ; \alpha_{\mathrm{S}}\right)=\delta_{g a} \delta(1-z)+\sum_{n=1}^{\infty}\left(\frac{\alpha_{\mathrm{S}}}{\pi}\right)^{n} C_{g a}^{(n)}(z) \\
H_{g}^{H}\left(\alpha_{\mathrm{S}}\right)=1+\sum_{n=1}^{\infty}\left(\frac{\alpha_{\mathrm{S}}}{\pi}\right)^{n} H_{g}^{H(n)}
\end{gathered}
$$

Since the partonic functions $G_{g a}$ and $C_{g a}$ are process independent, they fulfil the following relations:

$$
G_{g q_{f}}\left(z ; \alpha_{\mathrm{S}}\right)=G_{g \bar{q}_{f^{\prime}}}\left(z ; \alpha_{\mathrm{S}}\right) \equiv G_{g q}\left(z ; \alpha_{\mathrm{S}}\right), \quad C_{g q_{f}}\left(z ; \alpha_{\mathrm{S}}\right)=C_{g \bar{q}_{f^{\prime}}}\left(z ; \alpha_{\mathrm{S}}\right) \equiv C_{g q}\left(z ; \alpha_{\mathrm{S}}\right)
$$

which are a consequence of charge conjugation invariance and flavour symmetry of QCD. The dependence of $G_{g a}\left(C_{g a}\right)$ on the parton label $a$ is thus fully specified by $G_{g g}$ and $G_{g q}\left(C_{g g}\right.$ and $\left.C_{g q}\right)$. The first-order coefficient functions $G_{g g}^{(1)}(z)$ and $G_{g q}^{(1)}(z)$ (they are independent of the resummation scheme) are known [5]:

$$
G_{g g}^{(1)}(z)=C_{A} \frac{1-z}{z}, \quad G_{g q}^{(1)}(z)=C_{F} \frac{1-z}{z} .
$$

The first-order coefficient function $C_{g q}^{(1)}(z)$ is also independent on the resummation scheme; its expression is [27]

$$
C_{g q}^{(1)}(z)=\frac{1}{2} C_{F} z
$$

Using the large- $m_{t o p}$ approximation, the first-order coefficients $H_{g}^{H(1)}$ and $C_{g g}^{(1)}(z)$ fulfil the following relation [9]:

$$
C_{g g}^{(1)}(z)+\frac{1}{2} H_{g}^{H(1)} \delta(1-z)=\frac{\left(5+\pi^{2}\right) C_{A}-3 C_{F}}{4} \delta(1-z)
$$

The separate determination of $C_{g g}^{(1)}(z)$ and $H_{g}^{H(1)}$ requires the specification of a resummation scheme. For instance, considering the resummation scheme in which the SM Higgs boson coefficient $H_{g}^{H(1)}$ vanishes, the right-hand side of Eq. (15) gives the value of $C_{g g}^{(1)}(z)$ [27], and the corresponding value of the gluon form factor coefficient $B_{g}^{(2)}$ is explicitly reported in Eq. (128) of the second paper in Ref. [9]. The computation of the second-order coefficients $C_{g q}^{(2)}, C_{g g}^{(2)}$ and $H_{g}^{H(2)}$ is the aim of the calculation described in this paper.

For later purposes, we also define the following hard-collinear coefficient function:

$$
\mathcal{H}_{g g \leftarrow a b}^{H}\left(z ; \alpha_{\mathrm{S}}\right) \equiv H_{g}^{H}\left(\alpha_{\mathrm{S}}\right) \int_{0}^{1} d z_{1} \int_{0}^{1} d z_{2} \delta\left(z-z_{1} z_{2}\right)\left[C_{g a}\left(z_{1} ; \alpha_{\mathrm{S}}\right) C_{g b}\left(z_{2} ; \alpha_{\mathrm{S}}\right)+G_{g a}\left(z_{1} ; \alpha_{\mathrm{S}}\right) G_{g b}\left(z_{2} ; \alpha_{\mathrm{S}}\right)\right],
$$

which is directly related to the coefficient function in Eq. (8). There are only two differences between Eqs. (8) and (16). The first difference is due to the fact that the function $\mathcal{H}^{H}$ depends on the energy fraction $z$, since the right-hand side of Eq. (16) involves a convolution integral over the momentum fractions $z_{1}$ and $z_{2}$. This convolution kinematically arises by considering the 
integration of Eq. (7) over the rapidity $y$ of the Higgs boson. The second difference regards the scale of $\alpha_{\mathrm{S}}$ : in the functions $H^{H}\left(\alpha_{\mathrm{S}}\right), C\left(\alpha_{\mathrm{S}}\right)$ and $G\left(\alpha_{\mathrm{S}}\right)$ on the right-hand side of Eq. (16), the argument of $\alpha_{\mathrm{S}}$ is set to the same value (this common scale is not explicitly denoted in Eq. (16)). Owing to this feature, the process-dependent function $\mathcal{H}_{g g \leftarrow a b}^{H}$ is unambiguously defined (i.e., it is independent of the specification of the resummation scheme) [4]. The perturbative expansion of the function $\mathcal{H}^{H}$ directly follows from Eqs. (9)-(11). We have:

$$
\mathcal{H}_{g g \leftarrow a b}^{H}\left(z ; \alpha_{\mathrm{S}}\right)=\delta_{g a} \delta_{g b} \delta(1-z)+\sum_{n=1}^{\infty}\left(\frac{\alpha_{\mathrm{S}}}{\pi}\right)^{n} \mathcal{H}_{g g \leftarrow a b}^{H(n)}(z)
$$

where the first-order and second-order contributions are

$$
\begin{gathered}
\mathcal{H}_{g g \leftarrow a b}^{H(1)}(z)=\delta_{g a} \delta_{g b} \delta(1-z) H_{g}^{H(1)}+\delta_{g a} C_{g b}^{(1)}(z)+\delta_{g b} C_{g a}^{(1)}(z) \\
\mathcal{H}_{g g \leftarrow a b}^{H(2)}(z)=\delta_{g a} \delta_{g b} \delta(1-z) H_{g}^{H(2)}+\delta_{g a} C_{g b}^{(2)}(z)+\delta_{g b} C_{g a}^{(2)}(z)+H_{g}^{H(1)}\left(\delta_{g a} C_{g b}^{(1)}(z)+\delta_{g b} C_{g a}^{(1)}(z)\right) \\
+\left(C_{g a}^{(1)} \otimes C_{g b}^{(1)}\right)(z)+\left(G_{g a}^{(1)} \otimes G_{g b}^{(1)}\right)(z) .
\end{gathered}
$$

In Eq. (19) and in the following, the symbol $\otimes$ denotes the convolution integral (i.e., we define $\left.(g \otimes h)(z) \equiv \int_{0}^{1} d z_{1} \int_{0}^{1} d z_{2} \delta\left(z-z_{1} z_{2}\right) g\left(z_{1}\right) h\left(z_{2}\right)\right)$.

After our illustration of the all-order resummation formula in Eq. (7), we can return to its relation with the perturbative expression of the cumulative partonic cross section in Eq. (2). Using the Altarelli-Parisi evolution equations, the parton densities $f_{a / h}\left(x, b_{0}^{2} / b^{2}\right)$ on the right-hand side of Eq. (7) can be expressed in terms of the corresponding parton densities $f_{a / h}\left(x, M^{2}\right)$ at the factorization (evolution) scale $\mu_{F}=M$. Having done that, all the remaining factors in Eq. (7) are the partonic contributions that determine the small- $q_{T}$ singular component of the Higgs boson partonic cross section $d \hat{\sigma}_{a b} / d q_{T}^{2}$ in Eqs. (1) and (2). At fixed values of the impact parameter $b$, all these partonic contributions can be expanded in powers of $\alpha_{\mathrm{S}}\left(M^{2}\right)$, thus leading to perturbative coefficients that depend on powers of $\ln \left(b^{2} M^{2}\right)$. The dependence on $\ln \left(b^{2} M^{2}\right)$ is produced by the gluon form factor $S_{g}(M, b)$, by the Altarelli-Parisi evolution equations and by the QCD coupling ${ }^{\ddagger}$ $\alpha_{\mathrm{S}}\left(b_{0}^{2} / b^{2}\right)$. The powers of $\ln \left(b^{2} M^{2}\right)$ can then be transformed into logarithms, $\ln \left(M^{2} / q_{T}^{2}\right)$, in $q_{T^{-}}$ space by explicitly performing the Bessel transformation (i.e. the integration over $b$ ) in Eq. (7). This procedure, which involves manipulations that are standard in the context of transversemomentum resummation (technical details can be found, for instance, in Ref. [19]), yields the explicit perturbative expression of the singular component of $d \hat{\sigma}_{a b} / d \hat{y} d q_{T}^{2}$. The integration of this expression over $\hat{y}$ ( $\hat{y}$ is the rapidity of the Higgs boson in the centre-of-mass frame of the two colliding partons) and $q_{T}$ finally gives the cumulative partonic cross section of Eqs. (2) and (4) in the limit $Q_{0} \ll M$ (i.e., modulo the contributions of $\mathcal{O}\left(Q_{0}^{2} / M^{2}\right)$ in Eqs. (5) and (6)). The perturbative functions $\hat{R}_{a b}^{(n)}\left(z, M / Q_{0}\right)$ of Eq. (4) have a dependence on $l_{0}=\ln \left(M^{2} / Q_{0}^{2}\right)$ that is explicitly determined by the resummation formula (7), whereas the dependence on $z$ is given in terms of the $q_{T}$ resummation coefficients (those of the gluon form factor and in Eqs. (9)-(11)).

The NLO and NNLO functions $\hat{R}_{a b}^{(1)}$ and $\hat{R}_{a b}^{(2)}$ have the following expressions:

$$
\hat{R}_{a b}^{(1)}\left(z, M / Q_{0}\right)=l_{0}^{2} \Sigma_{g g \leftarrow a b}^{H(1 ; 2)}(z)+l_{0} \Sigma_{g g \leftarrow a b}^{H(1 ; 1)}(z)+\mathcal{H}_{g g \leftarrow a b}^{H(1)}(z)+\mathcal{O}\left(Q_{0}^{2} / M^{2}\right)
$$

${ }_{\ddagger}^{\ddagger}$ The coupling $\alpha_{\mathrm{S}}\left(b_{0}^{2} / b^{2}\right)$ can be expressed in terms of $\alpha_{\mathrm{S}}\left(M^{2}\right)$ and $\ln \left(b^{2} M^{2} / b_{0}^{2}\right)$ by using the renormalization group equation for the perturbative $\mu^{2}$-evolution of the running coupling $\alpha_{\mathrm{S}}\left(\mu^{2}\right)$. 


$$
\begin{aligned}
\hat{R}_{a b}^{(2)}\left(z, M / Q_{0}\right) & =l_{0}^{4} \Sigma_{g g \leftarrow a b}^{H(2 ; 4)}(z)+l_{0}^{3} \Sigma_{g g \leftarrow a b}^{H(2 ; 3)}(z)+l_{0}^{2} \Sigma_{g g \leftarrow a b}^{H(2 ; 2)}(z)+l_{0}\left(\Sigma_{g g \leftarrow a b}^{H(2 ; 1)}(z)-16 \zeta_{3} \Sigma_{g g \leftarrow a b}^{H(2 ; 4)}(z)\right) \\
& +\left(\mathcal{H}_{g g \leftarrow a b}^{H(2)}(z)-4 \zeta_{3} \Sigma_{g g \leftarrow a b}^{H(2 ; 3)}(z)\right)+\mathcal{O}\left(Q_{0}^{2} / M^{2}\right)
\end{aligned}
$$

which are consistent with the behaviour in Eqs. (5) and (6). In Eqs. (20) and (21) we use the same notation as in Ref. [19]. The coefficient functions $\Sigma_{g g \leftarrow a b}^{H(n ; m)}(z)$ depend on the $q_{T}$ resummation coefficients: the explicit expressions are given in Eqs. (63),(64),(66)-(69) of Ref. [19] (we have to set $\mu_{R}=\mu_{F}=Q=M$, where $\mu_{R}, \mu_{F}$ and $Q$ are the auxiliary scales of Ref. [19]) and are not reported here. The coefficients $\mathcal{H}_{g g \leftarrow a b}^{H(1)}$ and $\mathcal{H}_{g g \leftarrow a b}^{H(2)}$ are exactly those in Eqs. (18) and (19). The first-order terms $\Sigma_{g g \leftarrow a b}^{H(1 ; 2)}$ and $\Sigma_{g g \leftarrow a b}^{H(1 ; 1)}$ depend on the gluon form factor $S_{g}(M, b)$. The second-order terms $\Sigma_{g g \leftarrow a b}^{H(2 ; m)}$ depend on $\mathcal{H}_{g g \leftarrow a b}^{H(1)}$ and on the gluon form factor $S_{g}(M, b)$ up to $\mathcal{O}\left(\alpha_{\mathrm{S}}^{2}\right)$. The numerical coefficient $\zeta_{3} \simeq 1.202 \ldots$ ( $\zeta_{k}$ is the Riemann $\zeta$-function) on the right-hand side of Eq. (21) originates from the Bessel transformations (see, e.g., Eqs. (B.18) and (B.30) in Appendix B of Ref. [19]).

The relations (20) and (21) can be exploited in two different ways. From the knowledge of the perturbative coefficients of the resummation formulae (7) and (8), we can compute $\Sigma^{H(n ; m)}$ and $\mathcal{H}^{H(n)}$ and then, we can obtain a perturbative prediction for the cumulative partonic cross section up to NNLO. Alternatively, from the explicit NNLO perturbative computation of the cumulative partonic cross section, we can extract $\Sigma^{H(n ; m)}$ and $\mathcal{H}^{H(n)}$ and then, we can determine the $q_{T}$ resummation coefficients up to $\mathcal{O}\left(\alpha_{\mathrm{S}}^{2}\right)$. 
Our NNLO computation of the cumulative partonic cross section is described in the final part of this paper. We obtain the following results. The explicit result of the NLO function $\hat{R}_{a b}^{(1)}(z)$ confirms the expressions of $\Sigma_{g g \leftarrow a b}^{H(1 ; 2)}(z), \Sigma_{g g \leftarrow a b}^{H(1 ; 1)}(z)$ and $\mathcal{H}_{g g \leftarrow a b}^{H(1)}(z)$, as predicted by the $q_{T}$ resummation coefficients at $\mathcal{O}\left(\alpha_{\mathrm{S}}\right)$. At NNLO, the present knowledge $[8,9]$ of the $q_{T}$ resummation coefficients at $\mathcal{O}\left(\alpha_{\mathrm{S}}^{2}\right)$ predicts the expressions of the terms $\Sigma_{g g \leftarrow a b}^{H(2 ; m)}(z)$, with $m=1,2,3,4$. Our result for the NNLO function $\hat{R}_{a b}^{(2)}(z)$ confirms this prediction, and it allows us to extract the explicit expression of the second-order coefficient function $\mathcal{H}_{g g \leftarrow a b}^{H(2)}(z)$.

We obtain:

$$
\begin{aligned}
& \mathcal{H}_{g g \leftarrow q q}^{H(2)}(z)=-C_{F}^{2}\left[\frac{2(1-z)}{z}+\frac{(2+z)^{2}}{4 z} \ln z\right], \\
& \mathcal{H}_{g g \leftarrow g q}^{H(2)}(z)=C_{F}^{2}\left(\frac{1}{48}(2-z) \ln ^{3} z-\frac{1}{32}(3 z+4) \ln ^{2} z+\frac{5}{16}(z-3) \ln z\right. \\
& +\frac{1}{12}\left(\frac{1}{z}+\frac{z}{2}-1\right) \ln ^{3}(1-z)+\frac{1}{16}\left(z+\frac{6}{z}-6\right) \ln ^{2}(1-z) \\
& \left.+\left(\frac{5 z}{8}+\frac{2}{z}-2\right) \ln (1-z)+\frac{5}{8}-\frac{13}{16} z\right) \\
& +C_{F} n_{F}\left(\frac{1}{24 z}\left(1+(1-z)^{2}\right) \ln ^{2}(1-z)+\frac{1}{18}\left(z+\frac{5}{z}-5\right) \ln (1-z)\right. \\
& \left.-\frac{14}{27}+\frac{14}{27 z}+\frac{13}{108} z\right) \\
& +C_{F} C_{A}\left(-\frac{\left(1+(1+z)^{2}\right)}{2 z} \operatorname{Li}_{3}\left(\frac{1}{1+z}\right)+\left(\frac{1}{2}-\frac{5}{2 z}-\frac{5}{4} z\right) \operatorname{Li}_{3}(z)\right. \\
& -\frac{3}{4 z}\left(1+(1+z)^{2}\right) \operatorname{Li}_{3}(-z)+\left(2-\frac{11}{6 z}-\frac{z}{2}+\frac{z^{2}}{3}+\left(-\frac{1}{2}+\frac{3}{2 z}+\frac{3 z}{4}\right) \ln z\right) \operatorname{Li}_{2}(z) \\
& +\left(\frac{z}{4}+\frac{\left(1+(1+z)^{2}\right)}{4 z} \ln (z)\right) \operatorname{Li}_{2}(-z) \\
& +\frac{\left(1+(1+z)^{2}\right)}{12 z} \ln ^{3}(1+z)-\frac{1}{24 z}\left(\left(1+(1+z)^{2}\right)\left(3 \ln ^{2} z+\pi^{2}\right)-6 z^{2} \ln z\right) \ln (1+z) \\
& -\frac{\left.\left(1+(1-z)^{2}\right)\right)}{24 z} \ln ^{3}(1-z)+\frac{1}{48 z}\left(6\left(1+(1-z)^{2}\right) \ln z-5 z^{2}-22(1-z)\right) \ln ^{2}(1-z) \\
& +\frac{1}{72 z}\left(-152+152 z-43 z^{2}+6\left(-22+24 z-9 z^{2}+4 z^{3}\right) \ln z+9\left(1+(1-z)^{2}\right) \ln ^{2} z\right) \ln (1-z) \\
& -\frac{1}{12}\left(1+\frac{z}{2}\right) \ln ^{3} z+\frac{1}{48}\left(36+9 z+8 z^{2}\right) \ln ^{2} z+\left(-\frac{107}{24}-\frac{1}{z}+\frac{z}{12}-\frac{11}{9} z^{2}\right) \ln z \\
& \left.+\frac{1}{z}\left(4 \zeta_{3}-\frac{503}{54}+\frac{11}{36} \pi^{2}\right)+\frac{1007}{108}-\frac{\pi^{2}}{3}-\frac{5}{2} \zeta_{3}+z\left(\frac{\pi^{2}}{3}+2 \zeta_{3}-\frac{133}{108}\right)+z^{2}\left(\frac{38}{27}-\frac{\pi^{2}}{18}\right)\right) \text {, }
\end{aligned}
$$




$$
\begin{aligned}
& \mathcal{H}_{g g \leftarrow g g}^{H(2)}(z)=\left(\left(-\frac{101}{27}+\frac{7}{2} \zeta_{3}\right) C_{A}^{2}+\frac{14}{27} C_{A} n_{F}\right)\left(\frac{1}{1-z}\right)_{+} \\
& +\left(C_{A}^{2}\left(\frac{3187}{288}+\frac{7}{8} L_{t}+\frac{157}{72} \pi^{2}+\frac{13}{144} \pi^{4}-\frac{55}{18} \zeta_{3}\right)+C_{A} C_{F}\left(-\frac{145}{24}-\frac{11}{8} L_{t}-\frac{3}{4} \pi^{2}\right)\right. \\
& +\frac{9}{4} C_{F}^{2}-\frac{5}{96} C_{A}-\frac{1}{12} C_{F}-C_{A} n_{F}\left(\frac{287}{144}+\frac{5}{36} \pi^{2}+\frac{4}{9} \zeta_{3}\right) \\
& \left.+C_{F} n_{F}\left(-\frac{41}{24}+\frac{1}{2} L_{t}+\zeta_{3}\right)\right) \delta(1-z) \\
& +C_{A}^{2}\left(\frac{\left(1+z+z^{2}\right)^{2}}{z(1+z)}\left(2 \operatorname{Li}_{3}\left(\frac{z}{1+z}\right)-\operatorname{Li}_{3}(-z)\right)+\frac{2-17 z-22 z^{2}-10 z^{3}-12 z^{4}}{2 z(1+z)} \zeta_{3}\right. \\
& -\frac{5-z+5 z^{2}+z^{3}-5 z^{4}+z^{5}}{z(1-z)(1+z)}\left(\operatorname{Li}_{3}(z)-\zeta_{3}\right)+\operatorname{Li}_{2}(z) \frac{\ln (z)}{1-z} \frac{3-z+3 z^{2}+z^{3}-3 z^{4}+z^{5}}{z(1+z)} \\
& +\frac{\left(1+z+z^{2}\right)^{2}}{z(1+z)}\left(\ln (z) \operatorname{Li}_{2}(-z)-\frac{1}{3} \ln ^{3}(1+z)+\zeta_{2} \ln (1+z)\right) \\
& +\frac{1-z}{3 z}\left(11-z+11 z^{2}\right) \operatorname{Li}_{2}(1-z)+\frac{1}{12} z \ln (1-z)-\frac{1}{6} \frac{\ln ^{3}(z)}{1-z} \frac{\left(1+z-z^{2}\right)^{2}}{1+z} \\
& +\ln ^{2}(z)\left(\frac{\left(1-z+z^{2}\right)^{2}}{2 z(1-z)} \ln (1-z)-\frac{\left(1+z+z^{2}\right)^{2}}{2 z(1+z)} \ln (1+z)+\frac{25-11 z+44 z^{2}}{24}\right) \\
& +\ln (z)\left(\frac{\left(1+z+z^{2}\right)^{2}}{z(1+z)} \ln ^{2}(1+z)+\frac{\left(1-z+z^{2}\right)^{2}}{2 z(1-z)} \ln ^{2}(1-z)\right. \\
& \left.\left.-\frac{72+773 z+149 z^{2}+536 z^{3}}{72 z}\right)+\frac{517}{27}-\frac{449}{27 z}-\frac{380 z}{27}+\frac{835 z^{2}}{54}\right) \\
& +C_{A} n_{F}\left(\frac{1+z}{12} \ln ^{2}(z)+\frac{1}{36}(13+10 z) \ln (z)-\frac{z}{12} \ln (1-z)-\frac{83}{54}+\frac{121}{108 z}+\frac{55}{54} z-\frac{139}{108} z^{2}\right) \\
& +C_{F} n_{F}\left(\frac{1+z}{12} \ln ^{3}(z)+\frac{1}{8}(3+z) \ln ^{2}(z)+\frac{3}{2}(1+z) \ln (z)-\frac{1-z}{6 z}\left(1-23 z+z^{2}\right)\right),
\end{aligned}
$$

where $L_{t}=\ln \left(M^{2} / m_{\text {top }}^{2}\right)\left(m_{\text {top }}\right.$ is the pole mass of the top quark) and $\operatorname{Li}_{k}(z)(k=2,3)$ are the usual polylogarithm functions,

$$
\mathrm{Li}_{2}(z)=-\int_{0}^{z} \frac{d t}{t} \ln (1-t), \quad \operatorname{Li}_{3}(z)=\int_{0}^{1} \frac{d t}{t} \ln (t) \ln (1-z t)
$$

We comment on the Higgs boson results in Eqs. (22)-(24) and on the ensuing determination of the second-order coefficients $C_{g q}^{(2)}, C_{g g}^{(2)}$ and $H_{g}^{H(2)}$ in Eqs. (10) and (11).

Considering the dependence on the parton indices $a$ and $b$, the hard-collinear function $\mathcal{H}_{g g \leftarrow a b}^{H}$ is 'flavour blind', namely, it fulfils the relations

$$
\mathcal{H}_{g g \leftarrow q_{f} q_{f^{\prime}}}^{H}=\mathcal{H}_{g g \leftarrow q_{f} \bar{q}_{f^{\prime}}}^{H}=\mathcal{H}_{g g \leftarrow \bar{q}_{f} q_{f^{\prime}}}^{H}=\mathcal{H}_{g g \leftarrow \bar{q}_{f} \bar{q}_{f^{\prime}}}^{H} \equiv \mathcal{H}_{g g \leftarrow q q}^{H},
$$




$$
\mathcal{H}_{g g \leftarrow q_{f} g}^{H}=\mathcal{H}_{g g \leftarrow g q_{f}}^{H}=\mathcal{H}_{g g \leftarrow g \bar{q}_{f^{\prime}}}^{H}=\mathcal{H}_{g g \leftarrow \bar{q}_{f^{\prime}} g}^{H} \equiv \mathcal{H}_{g g \leftarrow g q}^{H} .
$$

These symmetry relations follows from Eq. (12) and from the convolution integral in Eq. (16) (the convolution integral implies that $\mathcal{H}_{g g \leftarrow a b}^{H}$ is symmetric with respect to the exchange $a \leftrightarrow b$ ). Therefore, the parton matrix $\mathcal{H}_{g g \leftarrow a b}^{H(2)}$ is completely specified by the three entries in Eqs. (22)-(24): the quark-quark function $\mathcal{H}_{g g \leftarrow q q}^{H(2)}$, the gluon-quark function $\mathcal{H}_{g g \leftarrow g q}^{H(2)}$ and the gluon-gluon function $\mathcal{H}_{g g \leftarrow g g}^{H(2)}$.

Using Eq. (19), in the quark-quark channel we have

$$
\mathcal{H}_{g g \leftarrow q q}^{H(2)}(z)=\left(C_{g q}^{(1)} \otimes C_{g q}^{(1)}\right)(z)+\left(G_{g q}^{(1)} \otimes G_{g q}^{(1)}\right)(z) .
$$

We see that the second-order coefficient function $\mathcal{H}_{g g \leftarrow q q}^{H(2)}(z)$ is fully determined by the $q_{T}$ resummation coefficients at $\mathcal{O}\left(\alpha_{\mathrm{S}}\right)$. Using the values of $G_{g q}^{(1)}$ and $C_{g q}^{(1)}$ in Eqs. (13) and (14), the expression on the right-hand side of Eq. (28) is in complete agreement with the result in Eq. (22). Therefore, our explicit computation of the NNLO partonic function $\hat{R}_{q q}^{(2)}$ represents a consistency check of the factorization formula (8) and of the value of $G_{g q}^{(1)}$, which were derived in the process-independent study of Ref. [5].

Considering the gluon-quark channel, Eq. (19) can be recast in the following form:

$$
C_{g q}^{(2)}(z)+\frac{1}{2} H_{g}^{H(1)} C_{g q}^{(1)}(z)=\mathcal{H}_{g g \leftarrow g q}^{H(2)}(z)-\frac{1}{2}\left(\mathcal{H}_{g g \leftarrow g g}^{H(1)} \otimes C_{g q}^{(1)}\right)(z)-\left(G_{g g}^{(1)} \otimes G_{g q}^{(1)}\right)(z),
$$

where we have used $\mathcal{H}_{g g \leftarrow g g}^{H(1)}(z)=H_{g}^{H(1)} \delta(1-z)+2 C_{g g}^{(1)}(z)$ (see Eq. (18)). The relation (29) can be used to determine $C_{g q}^{(2)}(z)$ from the knowledge of $\mathcal{H}_{g g \leftarrow g q}^{H(2)}$ and of the $q_{T}$ resummation coefficients at $\mathcal{O}\left(\alpha_{\mathrm{S}}\right)$ (in particular, the values of $G_{g g}^{(1)}$ and $G_{g q}^{(1)}$ affect the determination of $C_{g q}^{(2)}$ ). Inserting the first-order results of Eqs. (13)-(15) in Eq. (29), we explicitly have:

$$
C_{g q}^{(2)}(z)+\frac{1}{4} H_{g}^{H(1)} C_{F} z=\mathcal{H}_{g g \leftarrow g q}^{H(2)}(z)+C_{F}^{2} \frac{3}{8} z+C_{F} C_{A} \frac{1}{z}\left[(1+z) \ln z+2(1-z)-\frac{5+\pi^{2}}{8} z^{2}\right],
$$

where $\mathcal{H}_{g g \leftarrow g q}^{H(2)}$ is given in Eq. (23). Note that the right-hand side of Eq. (29) (or Eq. (30)) is resummation-scheme independent. Analogously to Eq. (15), the dependence of $C_{g q}^{(2)}$ on the resummation scheme is thus parametrized by the first-order coefficient $H_{g}^{H(1)}$ on the left-hand side of Eq. (30).

The process-independent coefficient function $C_{g g}^{(2)}(z)$ is obtained analogously to $C_{g q}^{(2)}(z)$. Considering the gluon-gluon channel, Eq. (19) gives:

$$
\begin{aligned}
2 C_{g g}^{(2)}(z) & +\delta(1-z)\left[H_{g}^{H(2)}-\frac{3}{4}\left(H_{g}^{H(1)}\right)^{2}\right]+\frac{1}{2} H_{g}^{H(1)} \mathcal{H}_{g g \leftarrow g g}^{H(1)}(z) \\
& =\mathcal{H}_{g g \leftarrow g g}^{H(2)}(z)-\frac{1}{4}\left(\mathcal{H}_{g g \leftarrow g g}^{H(1)} \otimes \mathcal{H}_{g g \leftarrow g g}^{H(1)}\right)(z)-\left(G_{g g}^{(1)} \otimes G_{g g}^{(1)}\right)(z),
\end{aligned}
$$

where the right-hand side is expressed in terms of resummation-scheme independent functions. 
Inserting Eqs. (13)-(15) in Eq. (31), we explicitly obtain:

$$
\begin{aligned}
2 C_{g g}^{(2)}(z) & +\delta(1-z)\left[H_{g}^{H(2)}-\frac{3}{4}\left(H_{g}^{H(1)}\right)^{2}+\frac{\left(5+\pi^{2}\right) C_{A}-3 C_{F}}{4} H_{g}^{H(1)}\right]+ \\
& =\mathcal{H}_{g g \leftarrow g g}^{H(2)}(z)-\delta(1-z)\left[\frac{\left(5+\pi^{2}\right) C_{A}-3 C_{F}}{4}\right]^{2}+C_{A}^{2} \frac{1}{z}[(1+z) \ln z+2(1-z)],
\end{aligned}
$$

where $\mathcal{H}_{g g \leftarrow g g}^{H(2)}$ is given in Eq. (24). We observe that $C_{g g}^{(2)}(z)$ includes a resummation-scheme dependent part that is simply proportional to $\delta(1-z)$. This part depends on $H_{g}^{H(1)}$ and $H_{g}^{H(2)}$. We also recall [4] that the resummation-scheme invariance relates $C_{g g}^{(2)}, H_{g}^{H(2)}$ and the third-order coefficient $B_{g}^{(3)}$ of the gluon form factor.

We conclude the paper by briefly describing the method that we have used to perform the NNLO analytic computation of the Higgs boson cross section in Eq. (2). The NNLO partonic calculation has to be carried out by using dimensional regularization to evaluate the QCD scattering amplitudes and their integration over the partonic phase space. In the framework of the large- $m_{t o p}$ approximation, the relevant partonic subprocesses are: the gluon fusion subprocess $g g \rightarrow H$ up to the two-loop level, the single-emission subprocesses $a b \rightarrow H+c$ up to the one-loop level, and the double-emission subprocesses $a b \rightarrow H+c_{1}+c_{2}$ at the tree level. The corresponding scattering amplitudes are known and have been used in the analytic calculations of two relevant Higgs boson observables: the NNLO total cross section $\hat{\sigma}_{a b}^{(\text {tot })}[28,29,30]$ and the NLO differential cross section $d \hat{\sigma}_{a b} / d \hat{y} d q_{T}^{2}[31,32]$ at large $q_{T}$. To perform our NNLO calculation, we take advantage of these available results: both observables are indeed computed up to relative order $\alpha_{\mathrm{S}}^{2}$ with respect to the Born level cross section $\sigma_{H}^{(0)}\left(\alpha_{\mathrm{S}}\right)$. We rewrite the $q_{T}$ integration in Eq. (2) as follows:

$$
\begin{aligned}
\int_{0}^{Q_{0}^{2}} d q_{T}^{2} \frac{d \hat{\sigma}_{a b}}{d q_{T}^{2}}\left(q_{T}, M, \hat{s} ; \alpha_{\mathrm{S}}\right) & \equiv \int_{0}^{+\infty} d q_{T}^{2} \frac{d \hat{\sigma}_{a b}}{d q_{T}^{2}}\left(q_{T}, M, \hat{s} ; \alpha_{\mathrm{S}}\right)-\int_{Q_{0}^{2}}^{+\infty} d q_{T}^{2} \frac{d \hat{\sigma}_{a b}}{d q_{T}^{2}}\left(q_{T}, M, \hat{s} ; \alpha_{\mathrm{S}}\right) \\
& =\hat{\sigma}_{a b}^{(\mathrm{tot})}\left(M, \hat{s} ; \alpha_{\mathrm{S}}\right)-\int_{Q_{0}^{2}}^{\infty} d q_{T}^{2} \int_{-\infty}^{+\infty} d \hat{y} \frac{d \hat{\sigma}_{a b}}{d \hat{y} d q_{T}^{2}}\left(\hat{y}, q_{T}, M, \hat{s} ; \alpha_{\mathrm{S}}\right)
\end{aligned}
$$

The cumulative partonic cross section over the range $0<q_{T}<Q_{0}$ is thus obtained by subtraction according to Eq. (33): we start from the total $\operatorname{cross~section~}^{\S} \hat{\sigma}_{a b}^{\text {(tot) }}$ and we subtract the contribution due to the $q_{T}$ cross section in the 'large'- $q_{T}$ region where $q_{T}>Q_{0}$ (in the context of Eq. (33), 'large' values of $q_{T}$ generically means 'non-vanishing' values of $q_{T}$ ). The differential cross section $d \hat{\sigma}_{a b} / d \hat{y} d q_{T}^{2}$ in the integrand on the right-hand side of Eq. (33) is presented in Ref. [31] in complete analytic form: we use this form and we explicitly carried out the integrations over $\hat{y}$ and $q_{T}$. Since $q_{T}>Q_{0}$, these integrations can directly be performed in four space-time dimensions, with no further use of dimensional regularization. At NLO, the cumulative partonic cross section can be computed in explicit analytic form for arbitrary values of $Q_{0}$, and the analytic result [33] is recalled below. At the NNLO, we limit ourselves to analytically computing the cumulative cross section in the limit $Q_{0} \ll M$, thus neglecting the terms of $\mathcal{O}\left(Q_{0}^{2} / M^{2}\right)$ on the right-hand side of Eqs. (6) or (21).

The NLO analytic result for the cumulative partonic cross section in Eqs. (2) and (33) was already presented in Ref. [33]. Indeed, at the NLO, the cumulative cross section exactly coincides

${ }^{\S}$ We actually use the expressions of Ref. [30], which are given for general colour factors, $C_{F}=\left(N_{c}^{2}-1\right) /\left(2 N_{c}\right)$ and $C_{A}=N_{c}$, of $S U\left(N_{c}\right)$. 
with the jet-vetoed cross section $\sigma^{\text {veto }}$ in Sect. 4 of Ref. [33], provided we identify $Q_{0}=p_{T}^{\text {veto }}$, where $p_{T}^{\text {veto }}$ is the jet veto parameter. Considering arbitrary values of $Q_{0}$, the NLO function $\hat{R}_{a b}^{(1)}\left(z, M / Q_{0}\right)$ of Eqs. (2) and (4) has the following form [33]:

$$
\hat{R}_{a b}^{(1)}\left(z, M / Q_{0}\right)=G_{a b}^{\mathrm{veto}(1)}\left(z ; \pi_{T}\right)=G_{a b}^{(1)(\mathrm{tot})}(z)-\Delta G_{a b}^{(1)}\left(z ; \pi_{T}\right) \Theta\left(1-\pi_{T}\right),
$$

where the variable $\pi_{T}$ depends on $z$ and $Q_{0} / M$,

$$
\pi_{T}=\pi_{T}\left(z, Q_{0} / M\right) \equiv \frac{2 Q_{0} \sqrt{z}}{(1-z) M}
$$

The two terms on the right-hand side of Eq. (34) are in one-to-one correspondence with the NLO contribution to the two terms on the right-hand side of Eq. (33). The partonic functions $\Delta G_{a b}^{(1)}\left(z ; \pi_{T}\right)$ are presented in Eq. (20) of Ref. [33], and $G_{a b}^{(1)(\text { tot })}(z)$ are the partonic functions of the NLO total cross section [34] $\left(G_{a b}^{(1)(\text { tot })}\right.$ is denoted by $G_{a b}^{(1)}$ in Ref. [33], and we have introduced the superscript '(tot)' to avoid confusion with the functions in our Eq. (13)). The explicit expressions of $G_{a b}^{(1)(\mathrm{tot})}(z)$ can be found in Eqs. (2.7)-(2.9) of Ref. [35], which uses the same overall normalization as in Eq. (34).

The NLO coefficient functions $\hat{R}_{a b}^{(1 ; k)}(z)(k=0,1,2)$ of Eq. (5) are obtained by performing the logarithmic expansion of the right-hand side of Eq. (34) at small values of $Q_{0}$. The limit $Q_{0} \ll M$ of Eq. (34) is not completely straightforward, since the functions $\hat{R}_{a b}^{(1 ; k)}(z)$ contain 'plus'distributions of the variable $z$. To illustrate this point we consider, for example, the complete expression of $\Delta G_{g g}^{(1)}\left(z ; \pi_{T}\right)$ in Eq. (20) of Ref. [33] and, neglecting terms that trivially vanish if $Q_{0} \rightarrow 0$, we obtain

$$
\begin{aligned}
& \Delta G_{g g}^{(1)}\left(z ; \pi_{T}\right) \Theta\left(1-\pi_{T}\right)=-C_{A} \frac{11(1-z)^{3}}{6 z} \\
& +2 \hat{P}_{g g}(z) \ln \left[\frac{(1-z) M}{2 \sqrt{z} Q_{0}}\left(1+\sqrt{1-\frac{4 Q_{0}^{2}}{M^{2}(1-z)^{2}}}\right)\right] \Theta\left(1-z-\frac{2 Q_{0}}{M}\right)+\mathcal{O}\left(\frac{Q_{0}^{2}}{M^{2}}\right),
\end{aligned}
$$

where $\hat{P}_{g g}(z)$ (see Eq. (21) in Ref. [33]) is the customary LO Altarelli-Parisi splitting function. The sole non-trivial point related to the limit $Q_{0} \rightarrow 0$ of Eq. (36) is due to the fact that $\hat{P}_{g g}(z)$ is proportional to $1 /(1-z)$ and, thus, singular when $z \rightarrow 1$. At finite values of $Q_{0}$ this singularity is screened by the $\Theta$-function in Eq. (36), and the limit $Q_{0} \rightarrow 0$ has to be properly treated by introducing customary (mathematical) distributions, such as $\delta(1-z)$ and ' + '-distributions (see, e.g., Eq. (9) in Ref. [33]), that act onto smooth functions defined over the range $0 \leq z \leq 1$. In the specific case of Eq. (36), these distributions occur in the following expressions:

$$
\begin{aligned}
& \frac{\ln ^{k}(1-z)}{1-z} \Theta\left(1-z-\frac{2 Q_{0}}{M}\right)=\left[\frac{\ln ^{k}(1-z)}{1-z}\right]_{+}+\frac{(-1)^{k}}{k+1} \ln ^{k+1}\left(\frac{M}{2 Q_{0}}\right) \delta(1-z)+\mathcal{O}\left(\frac{Q_{0}}{M}\right),(37) \\
& \frac{1}{1-z} \ln \left[\frac{1}{2}\left(1+\sqrt{1-\frac{4 Q_{0}^{2}}{M^{2}(1-z)^{2}}}\right)\right] \Theta\left(1-z-\frac{2 Q_{0}}{M}\right)=\left(-\frac{\pi^{2}}{24}+\frac{1}{2} \ln ^{2} 2\right) \delta(1-z)+\mathcal{O}\left(\frac{Q_{0}}{M}\right) .
\end{aligned}
$$

Inserting Eqs. (37) and (38) in Eqs. (36) and (34), we obtain the logarithmic expansions in Eqs. (5) and (20), and the reader can directly crosscheck the correct values of $\Sigma_{g g \leftarrow g g}^{H(1 ; 2)}(z), \Sigma_{g g \leftarrow g g}^{H(1 ; 1)}(z)$ (see Eqs. (63) and (64) in Ref. [19]) and $\mathcal{H}_{g g \leftarrow g g}^{H(1)}$ (see Eq. (84) in Ref. [19], or Eqs. (15) and (18) herein). 
Our explicit computation of the coefficient functions $\mathcal{H}_{g g \leftarrow a b}^{H(2)}$ and, more generally, of the NNLO function $\hat{R}_{a b}^{(2)}\left(z, M / Q_{0}\right)$ in Eq. (21) closely follows the same steps that we have just illustrated at the NLO. The details are too complicated and lengthy to present here. Using Eq. (33), we obtain the NNLO analogue of Eq. (34) and the corresponding partonic function $\Delta G_{a b}^{(2)}$ arises from the $q_{T}$ (and $y$ ) integration of the last term on the right-hand side of Eq. (33). We carry out this analytic integration by neglecting terms that trivially vanish if $Q_{0} \rightarrow 0$, and we obtain an NNLO analytic expression that is (conceptually) analogous to Eq. (36). The final step of the NNLO calculation is analogous to that in Eqs. (37) and (38), and it involves a proper treatment of the limit $Q_{0} \ll M$ for several functions that become singular at the endpoint $z=1$.

In this paper we have considered the production of the SM Higgs boson at hadron colliders. We have presented the NNLO analytic calculation of the cross section at small values of $q_{T}$ (see Eqs. (2) and (6)). The NNLO result is compared (see Eq. (21)) with the predictions of transverse-momentum resummation (see Eqs. (7) and (8)). The comparison gives a crosscheck of the factorization formula (8) (see also Eq. (28)) and allows us to determine the previously unknown resummation coefficients at $\mathcal{O}\left(\alpha_{\mathrm{S}}^{2}\right)$. These are the coefficient functions $\mathcal{H}_{g g \leftarrow a b}^{H(2)}(z)$ (see Eqs. (22)-(24)) and the related coefficients $C_{g q}^{(2)}$ and $C_{g g}^{(2)}$ (see Eqs. (30) and (32)), which control the dependence on the rapidity of the Higgs boson. These coefficients can be implemented in resummed calculations of the inclusive $q_{T}$ distribution at full NNLL accuracy. Using the method of Ref. [11], the same coefficients are necessary to perform the fully-exclusive perturbative calculation up to NNLO.

Acknowledgements. This work was supported in part by the Research Executive Agency (REA) of the European Union under the Grant Agreement number PITN-GA-2010-264564 (LHCPhenoNet, Initial Training Network).

\section{References}

[1] Y. L. Dokshitzer, D. Diakonov and S. I. Troian, Phys. Lett. B 79 (1978) 269, Phys. Rep. 58 (1980) 269; G. Parisi and R. Petronzio, Nucl. Phys. B 154 (1979) 427; G. Curci, M. Greco and Y. Srivastava, Nucl. Phys. B 159 (1979) 451.

[2] J. C. Collins and D. E. Soper, Nucl. Phys. B 193 (1981) 381 [Erratum-ibid. B 213 (1983) 545], Nucl. Phys. B 197 (1982) 446.

[3] J. C. Collins, D. E. Soper and G. Sterman, Nucl. Phys. B 250 (1985) 199.

[4] S. Catani, D. de Florian and M. Grazzini, Nucl. Phys. B 596 (2001) 299.

[5] S. Catani and M. Grazzini, Nucl. Phys. B 845 (2011) 297.

[6] J. Kodaira and L. Trentadue, Phys. Lett. B 112 (1982) 66, report SLAC-PUB-2934 (1982), Phys. Lett. B 123 (1983) 335.

[7] C. T. H. Davies and W. J. Stirling, Nucl. Phys. B 244 (1984) 337; C. T. H. Davies, B. R. Webber and W. J. Stirling, Nucl. Phys. B 256 (1985) 413.

[8] S. Catani, E. D’Emilio and L. Trentadue, Phys. Lett. B 211 (1988) 335. 
[9] D. de Florian and M. Grazzini, Phys. Rev. Lett. 85 (2000) 4678, Nucl. Phys. B 616 (2001) 247.

[10] T. Becher and M. Neubert, report HD-THEP-10-13 (arXiv:1007.4005 [hep-ph]).

[11] S. Catani and M. Grazzini, Phys. Rev. Lett. 98 (2007) 222002.

[12] S. Catani, L. Cieri, G. Ferrera, D. de Florian and M. Grazzini, Phys. Rev. Lett. 103 (2009) 082001.

[13] G. Bozzi, S. Catani, G. Ferrera, D. de Florian and M. Grazzini, Phys. Lett. B 696 (2011) 207.

[14] C. Balazs and C. P. Yuan, Phys. Lett. B 478 (2000) 192; Q. H. Cao, C. R. Chen, C. Schmidt and C. P. Yuan, report ANL-HEP-PR-09-20 (arXiv:0909.2305 [hep-ph]).

[15] E. L. Berger and J. w. Qiu, Phys. Rev. D 67 (2003) 034026, Phys. Rev. Lett. 91 (2003) 222003.

[16] G. Bozzi, S. Catani, D. de Florian and M. Grazzini, Phys. Lett. B 564 (2003) 65.

[17] A. Kulesza and W. J. Stirling, JHEP 0312 (2003) 056

[18] A. Kulesza, G. F. Sterman and W. Vogelsang, Phys. Rev. D 69 (2004) 014012.

[19] G. Bozzi, S. Catani, D. de Florian and M. Grazzini, Nucl. Phys. B 737 (2006) 73.

[20] G. Bozzi, S. Catani, D. de Florian and M. Grazzini, Nucl. Phys. B 791 (2008) 1.

[21] S. Mantry and F. Petriello, Phys. Rev. D 83 (2011) 053007.

[22] D. de Florian, G. Ferrera, M. Grazzini and D. Tommasini, JHEP 1111 (2011) 064.

[23] M. Grazzini, JHEP 0802 (2008) 043.

[24] J. R. Ellis, M. K. Gaillard and D. V. Nanopoulos, Nucl. Phys. B 106 (1976) 292; M. A. Shifman, A. I. Vainshtein, M. B. Voloshin and V. I. Zakharov, Sov. J. Nucl. Phys. 30 (1979) 711.

[25] M. Kramer, E. Laenen and M. Spira, Nucl. Phys. B 511 (1998) 523.

[26] K. G. Chetyrkin, B. A. Kniehl and M. Steinhauser, Phys. Rev. Lett. 79 (1997) 353, Nucl. Phys. B 510 (1998) 61.

[27] R. P. Kauffman, Phys. Rev. D 45 (1992) 1512; C. P. Yuan, Phys. Lett. B283 (1992) 395.

[28] R. V. Harlander and W. B. Kilgore, Phys. Rev. Lett. 88 (2002) 201801.

[29] C. Anastasiou and K. Melnikov, Nucl. Phys. B 646 (2002) 220.

[30] V. Ravindran, J. Smith and W. L. van Neerven, Nucl. Phys. B 665 (2003) 325, Nucl. Phys. B704 (2005) 332.

[31] C. J. Glosser and C. R. Schmidt, JHEP 0212 (2002) 016. 
[32] V. Ravindran, J. Smith and W. L. Van Neerven, Nucl. Phys. B 634 (2002) 247.

[33] S. Catani, D. de Florian and M. Grazzini, JHEP 0201 (2002) 015.

[34] S. Dawson, Nucl. Phys. B 359 (1991) 283; A. Djouadi, M. Spira and P. M. Zerwas, Phys. Lett. B264 (1991) 440.

[35] S. Catani, D. de Florian and M. Grazzini, JHEP 0105 (2001) 025. 